\title{
Linx
}

Revue des linguistes de l'université Paris X Nanterre

5 | 1994

La négation

\section{Aspects du comportement pragmatique de la négation}

Albert Hamm

\section{OpenEdition}

Journals

Édition électronique

URL : http://journals.openedition.org/linx/1203

DOI : 10.4000/linx.1203

ISSN : 2118-9692

Éditeur

Presses universitaires de Paris Nanterre

\section{Édition imprimée}

Date de publication : 1 juin 1994

Pagination : 171-175

ISSN : 0246-8743

\section{Référence électronique}

Albert Hamm, «Aspects du comportement pragmatique de la négation », Linx [En ligne], 5 | 1994, mis en ligne le 18 juillet 2012, consulté le 19 avril 2019. URL : http://journals.openedition.org/linx/1203 ; DOI : $10.4000 / \operatorname{linx} .1203$

Ce document a été généré automatiquement le 19 avril 2019.

Département de Sciences du langage, Université Paris Ouest 


\title{
Aspects du comportement pragmatique de la négation
}

\author{
Albert Hamm
}

Notre contribution se réclame de la définition la plus large possible de la pragmatique, par exemple de la formulation qu'a proposée O. Ducrot pour la pragmatique «linguistique» dans Le dire et le dit :

${ }^{1}$ Si l'on donne pour objet à la pragmatique l'action humaine en général, le terme de pragmatique du langage peut servir à désigner, dans cet ensemble de recherches, celles qui concernent l'action humaine accomplie au moyen du langage, en indiquant ses conditions et sa portée.

2 Plus qu'un ensemble de résultats validés, nous nous proposons de présenter des interrogations et des recherches en cours; d'où le choix d'une table-ronde et d'un discours pluriel grâce à L. Danon-Boileau et J.-P. Descles qui ont accepté, à partir de points de vue différents, de tenter avec moi cette entreprise de triangulation.

Il est maintenant généralement acquis - la confrontation des principales théories linguistiques sur le problème de la négation le montre à l'évidence - qu'une approche purement syntaxique ne saurait suffire, pas plus d'ailleurs qu'une approche exclusivement sémantique ou notionnelle; en effet, toute une série de contraintes sur la négation s'expliquent par la structuration des réseaux de relations entre notions, comme l'a par exemple fort bien montré T. Givon ${ }^{2}$, autant que par les relations complexes que la négation entretient avec la plupart des catégories grammaticales. Enfin, parallèlement à son statut d'opérateur syntactico-sémantique complexe, la négation fonctionne très souvent comme un opérateur de mise en relation entre explicite et implicite, et ceci bien au-delà du fonctionnement qu'assigne $\mathrm{O}$. Ducrot à un type de négation «polémique» qu'il décrit comme suit : ${ }^{3}$

Ici, le locuteur de «Pierre n'est pas intelligent» en s'assimilant à l'énonciateur E2 du refus, s'oppose non pas à un locuteur, mais à un énonciateur E1 qu'il met en scène dans son discours même et qui peut n'être assimilé à l'auteur d'aucun discours effectif.

formule que J. Moeschler résume de la manière suivante :4 
Tout énoncé négatif n'est pas la réfutation d'un dire, ni d'une pensée, mais tout énoncé négatif convoque, fictivement, un dialogue polémique. nous, résistent encore tant soit peu à l'analyse, pour illustrer toute la complexité des problèmes liés à une approche pragmatique de la négation et tenter de mesurer le chemin qu'il reste au linguiste à parcourir.

est fourni par un brochure trouvée dans la salle, qui présente une des variations sur le scénario lucky-unlucky en deux photos pleines pages de la campagne publicitaire des cigarettes Lucky Strike : le préfixe négatif UN- n'a, dans ce contexte, pas véritablement valeur de négation si ce n'est en ce qu'il sert à constituer le second terme d'une opposition, opposition qui, elle-même, n'a d'autre fonction, d'autre valeur illocutoire, que de permettre, sans citer la marque ou de présenter le produit, de convoquer un implicite discursif et de créer par ce biais une association d'idées destinée à contourner en toute légalité les interdictions liées à la loi Evin sur la publicité des marques de tabac et d'alcool.

Il est d'ailleurs intéressant de noter qu'une étude comparative que nous avions menée sur le fonctionnement et l'évolution de la négation dans un corpus de slogans publicitaires dans des hebdomadaires de langue française et anglaise ${ }^{5}$ nous avait permis de faire apparaître que, tant la fréquence très faible des formes de négation observées dans les publicités pour des marques de tabac, que leur apparition relativement tardive faisait de ce type de messages publicitaires une catégorie bien spécifique ; en particulier la négation y apparaissait différemment de la façon dont elle se manifestait, par exemple, dans des publicités pour des marques d'alcools.

7 Un second type d'exemples - relativement peu communs il est vrai - qui ne se satisfont pas d'une analyse simple du fonctionnement pragmatique de la négation, mais posent la question de la taille du contexte et de la nature des éléments situationnels à prendre en compte, nous est notamment fourni par les énoncés suivants :

La seule chose que j'ai dite - et j'en suis sûr ! - c'est que je n'ai jamais dit ce qu'on a dit que j'aurais dit! Rien ne peut donc autoriser quiconque de bonne foi à dire que j'ai dit ce que je n'ai pas dit, d'autant plus que je redis que ceux qui continuent à dire que j'aurais dit n'étaient pas présents au moment et à l'endroit où j'aurais dit ce que je n'ai pas dit. Voilà pourquoi je ne peux rien dire de plus par rapport à ce que j'ai déjà dit sur ce que je n'ai jamais dit. J'espère seulement que, dans le passé, même récent, aucun de ceux qui me reprochent aujourd'hui de dire ce que je n'ai pas dit n'a fait, sans le dire ce que j'ai dit que je n'avais pas dit.

(Michel Charasse à l'Assemblée nationale, cité par l'Evénement du Jeudi du 29/12/88)

Mr Brown is listed as President of the Cambridge Anarchists. He says he runs the anarchists and they have meetings in his room, but he's not president because, being anarchist, they don't have a president, but even if they did he would not be known as the president because he's not really an anarchist, just kind of politically interested and someone had to step forward and do the organizing.

(The Sunday Times Magazine 22/01/67)

Au-delà de l'effet produit par l'empilement de négations produites à toute une série de niveaux d'énonciation réels ou construits, le cadre proposé par O. Ducrot pour l'analyse «polyphonique» de la négation permet, pour l'essentiel, une interprétation satisfaisante des faits observés. Rappelons brièvement ce que celui-ci disait dans une première version de cette analyse : 6 
Je proposerai de décrire tout énoncé de la forme non-p comme accomplissement de

2 actes illocutionnaires: l'un est l'affirmation de $p$ par un énonciateur E1 s'adressant à un destinataire D1, l'autre est le rejet de cette affirmation, rejet attribué à un énonciateur E2 s'adressant à D2.

Dans une seconde version, ${ }^{7}$ nous n'avons plus affaire à 2 actes illocutionnaires attribués à des locuteurs réels mais à l'expression de "points de vue» assignés à des «énonciateurs», et plus ou moins pris en charge par le locuteur :

...le sens de l'énoncé, dans la représentation qu'il donne de l'énonciation, peut y faire apparaître des voix qui ne sont pas celles d'un locuteur. J'appelle «énonciateurs» ces êtres qui sont censés s'exprimer à travers l'énonciation, sans que pour autant on leur attribue des mots précis ; s'ils parlent, c'est seulement en ce sens que l'énonciation est vue comme exprimant leur point de vue, leur position, leur attitude, mais non pas, au sens matériel du terme, leurs paroles.

10 L'analyse fine de ces phénomènes, et de leur combinaison avec la négation, permet également d'expliquer en partie le fonctionnement d'échantillons d'argumentation relevés à l'occasion de la récente campagne présidentielle aux Etats-Unis, fonctionnement déjà repéré antérieurement et dont on pourrait décrire schématiquement la complexité croissante de la manière suivante :

- l'attaque frontale : le candidat $\mathrm{W}$ dit de son adversaire : « M. X a eu une liaison avec sa secrétaire» mettant celui-ci en position inconfortable d'avoir à démentir cette information, réelle ou inventée; en termes linguistiques, il s'agit d'une opération relativement simple de négation d'un posé, mais, sur le plan des relations interpersonnelles, nous sommes en présence d'une situation dans laquelle $\mathrm{W}$ apparaît comme le calomniateur ou, dans l'hypothèse où l'information est authentique, tout au moins comme celui par qui le scandale arrive.

- l'anticipation d'attaque frontale : le candidat $\mathrm{W}$ dit à propos de son adversaire: «je trouve scandaleux que $\mathrm{M}$. X puisse insinuer que j'ai eu une liaison avec ma secrétaire alors que c'est un mensonge éhonté ». X n'a bien évidemment rien affirmé de semblable et il se trouve ainsi conduit à démentir des propos calomniateurs qu'il n'a pas tenus et mis en position d'assumer le rôle ingrat du calomniateur, que $\mathrm{W}$ tenait auparavant; de plus, sur le plan linguistique, il s'agit dans ce cas de nier un présupposé, opération plus délicate.

- le degré suivant dans l'escalade pourrait être l'affirmation suivante de W : « M. X va vous dire que je vais l'attaquer sur sa liaison avec sa secrétaire, loin de moi cette idée. Avec moi il ne sera jamais question que des véritables enjeux de cette élection ». X n'a toujours rien dit de semblable, et il se trouve placé dans une situation où la réfutation est pratiquement impossible. En effet, le démenti devrait porter ici à la fois sur un posé, celui du discours rapporté non tenu ( «Je n'ai jamais dit / eu l'intention de dire que M. W allait m'attaquer...») et sur un présupposé ( «Je n'ai pas eu de liaison avec ma secrétaire ») qui, de toute manière, n'a pas été pris en charge par W. Ce présupposé correspond, en bonne analyse polyphonique, à l'attribution par $\mathrm{W}$ à X d'un point de vue, point de vue que $\mathrm{X}$ lui-même est supposé, si l'on en croit $\mathrm{W}$, avoir attribué à W...

Nous avons également développé ailleurs, ${ }^{8}$ à propos de l'analyse de quelques formes de constructions négatives intéressantes rencontrées dans The World According to Garp, de John Irving, l'esquisse d'une analyse métadiscursive, mettant en relation analyse polyphonique et logique narrative. 


\section{NOTES}

1. O. Ducrot, Le dire et le dit, Minuit, Paris 1984, p. 173.

2. T. Givon, On Understanding Grammar, Academic Press, New-York 1979.

3. O. Ducrot, op. cit., p. 217.

4. J. Moeschler, Les aspects pragmatiques de la négation linguistique: acte de langage, argumentation et inférence pragmatique, Travaux du Centre de Recherches Sémiologiques, $\mathrm{n}$

•59, Neuchâtel 1991, p. 124.

5. A. Hamm, Statut et fonctionnement de la négation en anglais contemporain, Doct. Et. Paris 1988.

6. O. Ducrot et al., Les mots du discours, Minuit, Paris 1980, p. 49.

7. O. Ducrot, op. cit., 1984, p. 204.

8. A. Hamm, « Vers une pragmatique de la négation », RANAM n XXIII, Strasbourg 1990.

\section{RÉSUMÉS}

La négation apparaît comme un opérateur syntactico-sémantique complexe. En témoignent en particulier les contraintes distributionnelles très fortes qui accompagnent sa présence, et le statut présupositionnel particulier des énoncés négatifs. Elle fonctionne également comme un opérateur de mise en relation entre explicite et implicite bien au-delà, par exemple, du fonctionnement qu'assigne $\mathrm{O}$. Ducrot à un type de négation 'polémique' L'étude de quelques exemples particuliers vise à illustrer la difficulté d'une analyse du fonctionnement pragmatique de la négation.

Negation appears to have a complex status as syntactico-semantic operator; evidence of this is provided by the various distributional restrictions which accompany its presence and by the fact that negative utterances are presuppositionally more marked than the corresponding affirmative utterances. Negation also functions as an operator signalling the existence of an implicit content, on a much broader basis than the uses O. Ducrot has evidenced of a 'polemic' negation. It is proposed here to illustrate through a few examples the difficulty of achieving a satisfactory description of the pragmatic dimension of negation.

\section{AUTEUR}

\section{ALBERT HAMM}

Strasbourg II 